\title{
Thermogravimetric Analysis and Stability Test of Fluoroalkylsilanes Grafted on Alumina Hollow Fibre Membranes
}

\author{
Sirichai Koonaphapdeelert ${ }^{1}, \mathrm{Z} . \mathrm{Wu}^{2} \& \mathrm{~K} . \mathrm{Li}^{3}{ }^{3}$ \\ ${ }^{1}$ Department of Environmental Engincering, Chiang Mai University, Chiang Mai, 50200 Thailand \\ ${ }^{2 \& 3}$ Department of Chemical Engineering \& Chemical Technology, Imperial College London, London, SW7 2AZ \\ United Kingdom
}

\begin{abstract}
Alumina hollow tibre membrane surface were modified from hydrophilic to hydrophobic using fluoroalikylsilane (FAS) grafting technique which was found to be effective as shown by the increased contact angle (CA) from less than $90^{\circ}$ to more than $100^{\circ}$. The CA increased with respect to the grafting time and leveled off after 24 hours of grafting. The thermogravimetric analysis (TGA) showed that the FAS attached to the membranes were approximately $0.05-0.6 \%$ of the original membrane weight depending on the grafting time and the amount of hydroxyl groups present on the membrane substrates. According to the TGA result, the grafted membranes were thermally stable up to $250^{\circ} \mathrm{C}$ where FAS began to decompose. Then the grafted membranes were used as a contacting media for stripping $\mathrm{CO}_{2}$ off $2.5 \mathrm{M}$ monoethanolamine (MEA) solution at $80-100^{\circ} \mathrm{C}$. The membrancs were in contact with hot and corrosive MEA solution for approximately 100 hours without any operational problem. The scanning electron microscopy of the membrane surface showed no difference between the membrane before and after the operation. Also, the CAs of the grafted membranes remained unchanged. These findings suggest that FAS grafted membranes are chemically and thermally stable and can be used in membrane contactors at high temperatures and in chemically harsh conditions.
\end{abstract}

Keywords: Hollow fiber membrane, fluoroalkylsilanes, thermogravimetric analysis, stability test

\subsection{INTRODUCTION}

Although ceramic membranes, e.g, alumina, titania and zirconia membranes, are well known for their excellent thermal and chemical stabilities, they cannot be readily used in a membrane contactor since they are hydrophilic in nature and their pores can get wet very easily when contacting with aqueous solutions. Membrane wetting is a major problem in a membrane contacting process since it increases the mass transfer resistance in the membrane [1]. Thus the surface modification of ceramic membrane surfaces to promote their hydrophobicity has been developed in order to prevent the

\footnotetext{
$\overline{\text { * Corresponding to }}$ K. Li (emaiI; kang.li@imperial.ac.ulk)
}

membrane wetting problem. Among various modification techniques, silylation, which replaces an acidic hydrogen on the surface with a hydrophobic alkylsilyl group, is one of the most important and has drawn much research interest recently [2-4]

Fluoroalkylsilanes (FAS) is a common silylating agent used for ceramic surface modification and is found to be effective in hydrophobicity enhancement. Several studies on the silylating process and parameters have been conducted. Larbot et al. [5] modified tubular membranes made from zirconia and $\gamma$-alumina using fluoroalkylsilanes of different chain lengths. Hydrophobic membranes with high contact angles, $\left(\mathrm{CAs}>115^{\circ}\right)$ were obtained and the hydrophobic layers were thermodynamically stable up to $230^{\circ} \mathrm{C}$. Ceramic membrane distillation for 
desalination was also demonstrated using the grafted membranes and high salt rejection rates were observed. Akamatsu et al. [6] applied the polymerization of FAS on glass to adopt a waterrepellent feature and studied the polymerization condition as well as the layer durability.

Picard et al. [7] modified their ownmade zirconia membranes with trifunctional fluoroalkylsilanes having different lengths of hydrophobic tails $\left(\mathrm{C}_{\mathrm{n}} \mathrm{F}_{2 \mathrm{n}+1} \mathrm{C}_{2} \mathrm{H}_{4} \mathrm{Si}(\mathrm{OR})_{3}\right.$ where $\mathrm{n}=1,6,8, \mathrm{R}=\mathrm{OCH}_{3}, \mathrm{OC}_{2} \mathrm{H}_{5}$ ). The modified membrane showed high hydrophobicity as the liquid permeability significantly decreased, compared to the original membranes. Grafting time was found to be the main factor influencing the changes in hydrophobicity. The same authors also applied the FAS-modified zirconia and titania membranes to ozonation in wastewater treatment and obtained a good transfer rate with some advantages over the conventional contacting methods [8].

Krajewski et al. [9] studied the conditions of gralting, including the ratio of FAS per zirconia mass, grafting time and temperature. $1 \mathrm{H}, 1 \mathrm{H}, 2 \mathrm{H}, 2 \mathrm{H}$-perfluorodecyltriethoxysilane in chlorolorm was a grafiting solution. The result of thermogravimetric analysis (TGA) showed the optimum temperature at $30^{\circ} \mathrm{C}$, the ratio of FAS per zirconia at $1.429 \mathrm{mmol} / \mathrm{g}$ and the grafting time of 144 hours.

Koonaphapdeelert et al. suggested that alumina hollow fibre membranes prepared from a single-step fabrication can also be modified by FAS grafting [10]. Such membranes can be effectively used in membrane contactors for amine stripping at high temperature, the condition in which polymeric membranes cannot withstand [11]. However, the effects of grafting parameters on alumina hollow fibre substrates have never been reported before. Also, the membrane stability in the operation at high temperature has not been reported yet. In this study, the effects of grafting parameters, i.e. grafting time and ceramic substrates, were investigated through thermogravimetry. The thermal and chemical stabilities of the grafted membranes in an amine stripping process at high temperature were also examined.

\subsection{EXPERIMENTAL}

\subsection{Membrane Preparation}

Hollow fibre membranes were prepared from aluminium oxides using a phase inversion / sintering method. The alumina content of a spinning suspension was maintained at $60 \% \mathrm{w} / \mathrm{w}$ while polyethersulfone was used a binder. The membrane precursors were then sintered at different temperatures, ranged from $1200^{\circ} \mathrm{C}$ to $1500^{\circ} \mathrm{C}$. The details of ceramic membrane preparation method can be found elsewhere [10]. Membrane samples of $50 \mathrm{~mm}$ length from each batch were rinsed with DI water to remove any surface contaminants, dried in an oven at $100^{\circ} \mathrm{C}$ overnight and then be ready for surface modification. Some basic properties of the membranes are presented in Table 1.

Table 1 Basic properties of alumina hollow fibre membranes

\begin{tabular}{|c|c|c|c|c|c|c|}
\hline $\begin{array}{c}\text { Sintering } \\
\text { Temp. }\end{array}$ & $\begin{array}{c}\text { Outer } \varnothing \\
(\mathbf{m m})\end{array}$ & $\begin{array}{c}\text { Inner } \varnothing \\
\mathbf{( m m )}\end{array}$ & $\begin{array}{c}\text { Bending } \\
\text { Strength } \mathbf{( M P a )}\end{array}$ & $\begin{array}{c}\text { Effective } \\
\text { Porosity } \\
\left(\mathbf{m}^{-1)}\right.\end{array}$ & $\begin{array}{c}\text { Mean Pore } \\
\text { Radius }(\mu \mathbf{m})\end{array}$ & $\begin{array}{c}\text { SD of } \\
\text { pore } \\
\text { radius }\end{array}$ \\
\hline $1200^{\circ} \mathrm{C}$ & 1.47 & 1.02 & 18.2 & 14378 & 0.053 & 1.47 \\
\hline $1300^{\circ} \mathrm{C}$ & 1.40 & 0.97 & 35.5 & 11032 & 0.045 & 1.67 \\
\hline $1400^{\circ} \mathrm{C}$ & 1.28 & 0.89 & 80.8 & 4522 & 0.039 & 1.32 \\
\hline $1500^{\circ} \mathrm{C}$ & 1.14 & 0.78 & 183.1 & 1400 & 0.036 & 1.31 \\
\hline
\end{tabular}

*The effective porosity is defined as the ratio between the volumetric porosity of the membrane $(\varepsilon)$ and the membrane pore length (Lp), measured by the method proposed by Kong and Li [12] 


\subsection{Surface Modification}

The hollow fibre samples were immersed into the $0.01 \mathrm{~mol} /$ litre $1 \mathrm{H}, 1 \mathrm{H}, 2 \mathrm{H}, 2 \mathrm{H}$ perfluorooctylethoxysilane (FAS) (Lancaster synthesis) in n-hexane (Sigma Aldrich) solution in a vertically long glass tube at room temperature. The grafting time was varied from $0.5,2,8,24$, 48 and 72 hours to allow the coupling reaction between FAS and the ceramic surfaces to occur. After the immersion, the fibres were rinsed with n-hexane five times to remove any unreacted chemicals from the surfaces and dried at $100^{\circ} \mathrm{C}$ for 12 hours. Finally, the membranes were stored at room temperature and were ready for characterization.

\subsection{Membrane Characterization}

The membrane hydrophobicity was characterized by contact angle (CA) measurement using deionised (DI) water as testing liquid. The method used in this study was the Wilhemy method which is widely used to test the dynamic contact angles of samples which have different shapes other than flat sheet [13]. A hollow fibre sample was sealed off the opening end with an epoxy resin and hung on the microbalance in a tensiometer (Kruss K100). Then it was gradually immersed into the still testing-liquid. The rate of immersion was controlled at $6 \mathrm{~mm} / \mathrm{min}$ and all measurement was done at $20^{\circ} \mathrm{C}$. Three replicates were used in each measurement.

Thermogravimetric analysis (TA Instruments TGA-2050) was conducted to quantify the amount of FAS grafted onto the membranes. It was also used to determine decomposing temperature of FAS which reflected the membrane thermal stability. In each measurement, a small piece of membrane was placed on a platinum pan hung on a microbalance in an air-filled chamber. Then the temperature was increased at a rate of $10^{\circ} \mathrm{C} /$ minute to reach the maximum temperature at $600^{\circ} \mathrm{C}$. The weight of the sample was recorded on a real-time basis.

The micro-scale images of the membranes were obtained using scanning electron microscopy, SEM (JEOL JSM-5610LV). All samples were sputtered with gold and analyzed with a voltage of $15 \mathrm{KV}$. Outer and inner membrane surfaces were examined to reveal membrane structure and morphology.

\subsection{High Temperature Operation}

In order to test the chemical and thermal stabilities of the membranes, a number of surface modified membranes were packed into $1 / 2 " \varnothing$ stainless steel modules. The gaps among the fibres at both ends were sealed off using a special solvent-resistant epoxy resin. The modules were then used as packed columns for amine stripping at 80-100 ${ }^{\circ} \mathrm{C}$. MEA of $2.5 \mathrm{M}$ concentration was employed as the liquid absorbent in this study because it is a typical solvent for $\mathrm{CO}_{2}$ absorption and is reported to have corrosive effects on polymeric membranes [14-16]. The experinental setup of amine stripping unit is present in Figure 1. The details of each experimental run can be found elsewhere [11]. After approximately 100 hours of operation, the membranes samples were taken from the module and were characterized in order to examine any change occurred to the grafted membranes.

\subsection{RESULTS AND DISCUSSION}

\subsection{Membrane Hydrophobicity}

The CA of DI water on a membrane surface is a typical indicator of surface hydrophobicity. If the CA is less than $90^{\circ}$, the surface is considered hydrophilic. On the other hand, the surface with CA equal to $90^{\circ}$ or more is hydrophobic. In this study, the CA of deionised water on the unmodified alumina hollow fibre membranes were in a range of $70^{\circ}-80^{\circ}$, as shown in Figure 2. After just half an hour of surface grafting, the CA of water on the FAS grafted membranes increased to $90^{\circ}-110^{\circ}$, indicating the membrane surfaces were changed to hydrophobic. The CAs constantly increased as the grafting proceeded and leveled off after 24 hours of grafting. The maximum CAs, in a range of $110^{\circ}-130^{\circ}$, was showing fairly high hydrophobicity of the surfaces. The CAs obtained in this study were lower than the values reported in the literature [24-26], which were in a range of $140-150^{\circ} \mathrm{C}$, because the membranes in other studies were coated with $\gamma$-alumina which has a 


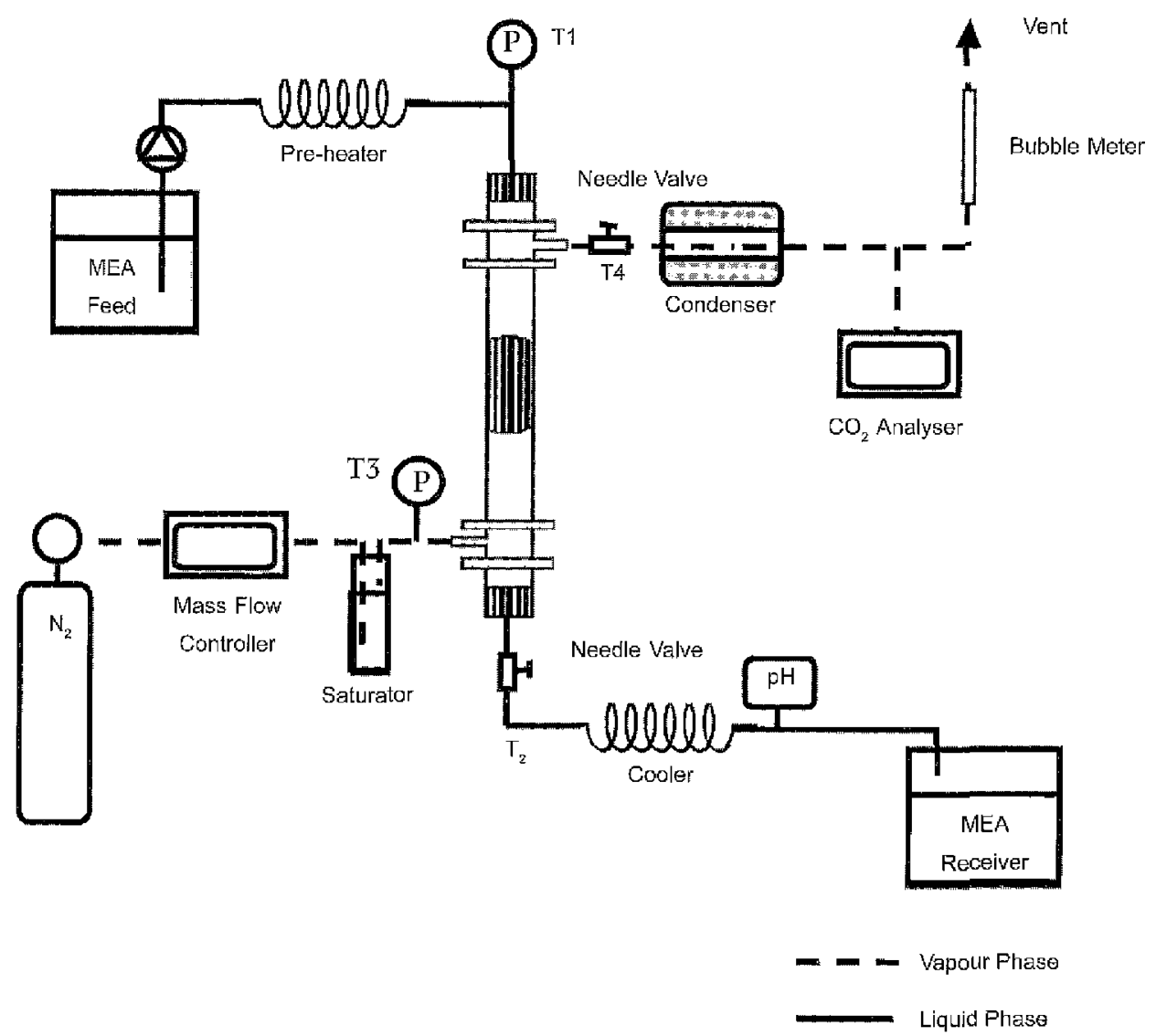

Figure 1 The experimental setup for $\mathrm{CO}_{2}$ stripping in a hollow fibre module ( $\mathrm{T}=$ thermocouples, $\mathrm{pH}=\mathrm{pH}$ meter, and $\mathrm{P}=$ pressure gauges)

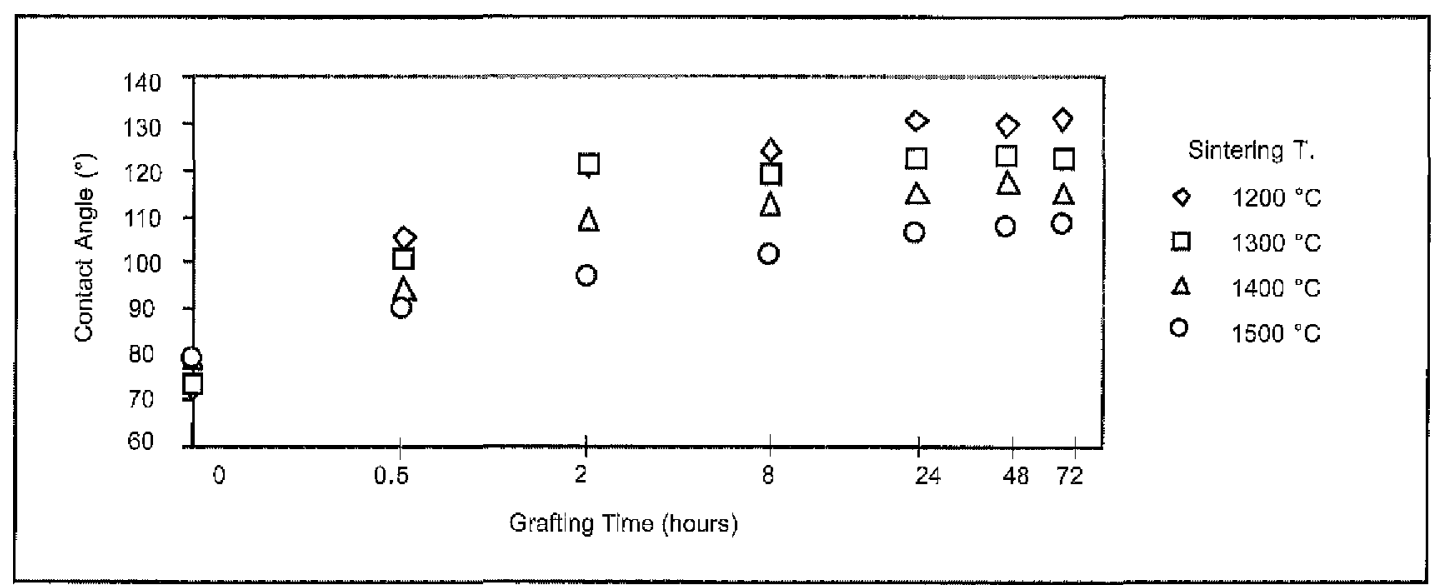

Figure 2 The contact angles of DI water on the membranes sintered at different temperatures and grafted for a different number of hours 
higher number of hydroxyl groups. However, the membrane preparation method used in this study was relatively simple as both $\gamma$ - and $\alpha$-alumina were mixed and spun at one time, compared to the other works in which the membranes were prepared by multi-step $\gamma$-alumina coating techniques. Such preparation simplicity is essential for the cost reduction of ceramic membranes which are often found too expensive in practical uses and commercialization

In Figure 2, the membranes sintered at different temperatures variedly responded to the surface modification. Those sintered at $1200^{\circ} \mathrm{C}$ had much higher CAs than those sintered at $1500^{\circ} \mathrm{C}$. This can be explained by that membranes sintered at $1200^{\circ} \mathrm{C}$ had more hydroxyl groups on the surface because alumina had not fully transformed from hydrous forms ( $\gamma$-alumina) to anhydrous form (a-alumina).

The effect of surface modification can be visually demonstrated in Figure 3. Short sections of grafted and non-grafted ceramic hollow fibres sintered at $1400^{\circ} \mathrm{C}$ were snapped off and freely dropped on the water surface. As can be seen, the surface-modified fibre was floating on the water surface because its contact angle was so high that water could not penetrate into the pores. As a result, the water surface tension could support the weight of the fibre. On the other hand, the nongrafted fibres immediately sank to the bottom of the beaker because the water broke into the pores right away and filled the voids inside.

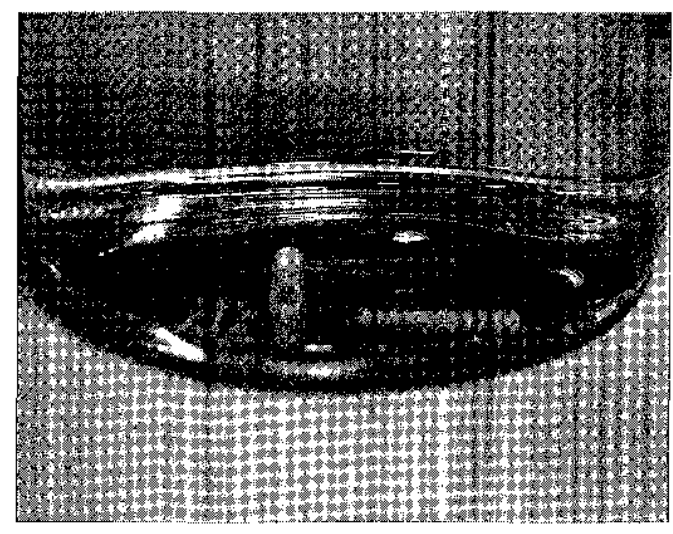

Grafted fibre

\subsection{Thermogravimetric Analysis}

The CA measurement in Section 3.1 shows that alumina membranes can be effectively modified to be hydrophobic. In this section, TGA was conducted to quantify the amount of FAS grafted on the membrane surface. Figure 4 shows the weight change profiles of membranes sintered at $1200^{\circ} \mathrm{C}$ and grafted with FAS for $0.5,2,8,24$, 48 and 72 hours, respectively. As can be seen, all the weight change profiles were in a reversed-S shape. As the temperature rose from $50^{\circ} \mathrm{C}$ to $250^{\circ} \mathrm{C}$, the membrane samples lost their weight due to the evaporation of moisture and easily-volatile contaminants. Then the samples began to lose their weight rapidly at approximately $250^{\circ} \mathrm{C}$ at which the silane compounds began to disassociate from the ceramic surfaces and decomposed. The temperature which had the maximum slope $\left(\mathrm{T}_{\max }\right)$ was $357^{\circ} \mathrm{C}$. This result is in line with the works by Picard et al. [7] and McElwee et al. [17] which reported the decomposing temperature of FAS at around $250^{\circ} \mathrm{C}$. Therefore, the maximum operating temperature of the membrane in any application. would be just below $250^{\circ} \mathrm{C}$.

Assuming that the FAS was completely burnt out at $600^{\circ} \mathrm{C}$, the amount of FAS grafted onto the membranes can be calculated based on the total weight loss of the membrane. Obviously the mass of FAS attached to the membranes depended on the grafting time. The grafted FAS weight gained to approximately $0.2 \%$ of the membrane weight

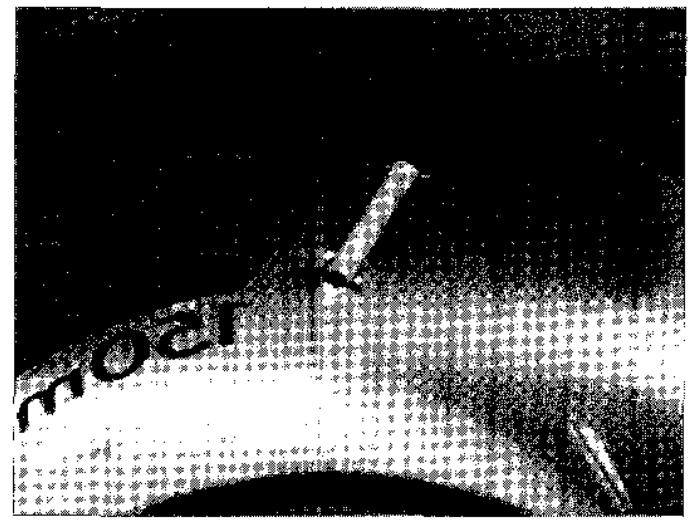

Non-grafted fibres

Figure 3 A short section of grafted fibre was floating on water whereas the original fibre sank 


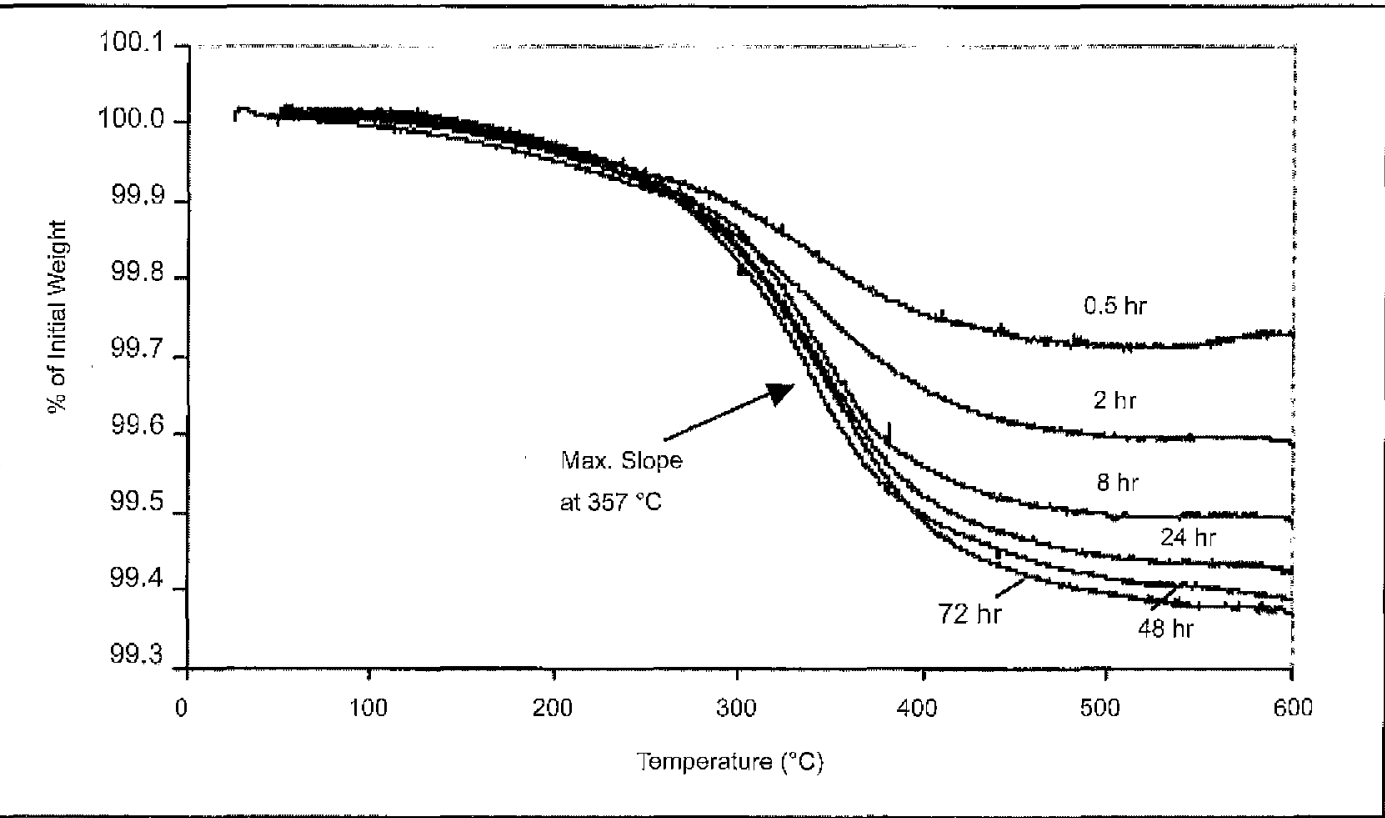

Eigure 4 The weight change profiles of the membranes sintered at $1200{ }^{\circ} \mathrm{C}$ and grafted with FAS for different periods of time

after just 0.5 hour of grafting time. The grafted FAS weight reached $0.4 \%, 0.5 \%$ and $0.6 \%$ after 2 , 8 and 24 hours of grafting, respectively. However, the grafting rate was slower as time passed since there were less hydroxyl sites available for the reaction. After 24 hours, the grafting was going on at a very slow rate as the weight gain was less than $0.1 \%$ even 72 hours had passed. At this point, almost all hydroxyl groups were expected to be occupied and the weight gain was a result of FAS polymerization.

Figure 5 shows the weight change profiles of the membranes sintered at different temperatures and grafted for 2 hours. As can be seen, the weight loss of the grafted membranes sintered at $1200{ }^{\circ} \mathrm{C}$ was approximately $0.6 \%$ of initial weight. The weight losses of the membranes sintered at higher temperatures, i.e. 1300,1400 and $1500{ }^{\circ} \mathrm{C}$, were decreased. This result can be explained by that there is a smaller number of hydroxyl groups available for the reaction on the membranes sintered at higher temperatures. The result also agrees with the result of CA measurennent in Section 3.1.
The amount of FAS grafted on the membrane sintered at $1500^{\circ} \mathrm{C}$ was merely $<0.05 \%$ of the total membrane weight, indicating that the grafting was not very effective. As the result, the CA of water on such membrane was just above $100^{\circ}$, near the border line of hydrophobicity at $90^{\circ}$. Therefore, membranes sintered at $1500^{\circ} \mathrm{C}$ were not appropriate for membrane contactors as their hydrophobicity was too low. Only the membranes sintered at $1200^{\circ} \mathrm{C}, 1300^{\circ} \mathrm{C}$ and $1400^{\circ} \mathrm{C}$ were used in the membrane stability test.

\subsection{Membrane Stability}

The thermal and chemical stabilities of the grafted membranes were investigated by testing them in a $\mathrm{CO}_{2}$ stripping experiment at high temperature. The hollow fibre membranes acted as contacting media that provided contacting area between hot gas and liquid. It was found that the menbranes sintered at $1200^{\circ} \mathrm{C}$ and $1300^{\circ} \mathrm{C}$ were unable to survive in the course of experiment because they were broken after a few hours of operation. Only the membranes sintered at $1400^{\circ} \mathrm{C}$ could withstand the 


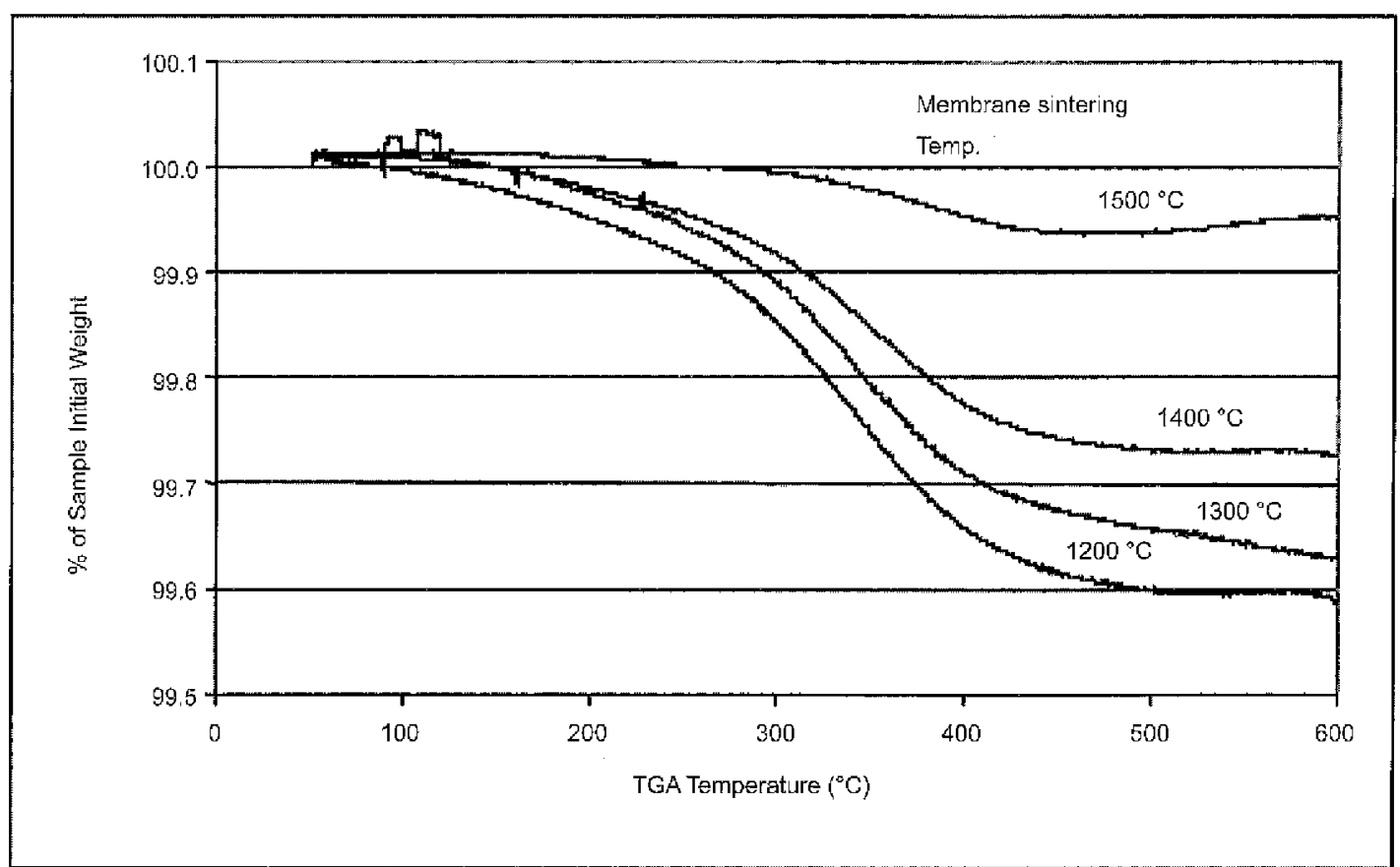

Figure 5 The weight change profiles of R10 membranes sintered at different temperatures and grafted for 2 hours

mechanical stress caused by material expansion at high temperature. Thus the membrane mechanical strength is very crucial for practical membrane application. The bending strength of the membrane sintered at $1400^{\circ} \mathrm{C}$ was $80.8 \mathrm{MPa}$ which can be regarded as the minimum strength required for this application.

After approximately 100 hours of operation, the membrane modules were disassembled and the membranes were taken out for inspection. Each sample was washed by deionised water and dried in an oven overnight. CA measurement was then performed on the samples. Table 2 shows the CA of the membranes with DI water. As can be seen, the CAs of the membranes after operation were slightly different but still within the standard deviation, indicating that the degree of hydrophobicity of the membranes remained unchanged after being exposed to high temperature MEA solution. Figure 6 shows the SEM micrographs of the nembrane surfaces before and after amine stripping experiment. As can be seen, their surface morphology was
Table 2 The contact angles of the membranes before and after operation

\begin{tabular}{|c|c|c|}
\hline \multirow{2}{*}{ Membranes } & \multicolumn{2}{|c|}{$\begin{array}{c}\text { Contact Angles with Water } \\
\text { (degrees) }\end{array}$} \\
\cline { 2 - 3 } & $\begin{array}{c}\text { Before } \\
\text { Operation }\end{array}$ & $\begin{array}{c}\text { After } \\
\text { Operation }\end{array}$ \\
\hline Module 1 & $115.1 \pm 2.8$ & $116.3 \pm 3.1$ \\
\hline Module 2 & $118.3 \pm 0.7$ & $116.9 \pm 1.9$ \\
\hline Module 3 & $115.1 \pm 2.8$ & $114.2 \pm 2.4$ \\
\hline
\end{tabular}

hardly changed. Thus it can be concluded that the modified membranes were fairly stable under the harsh operating condition.

The membranes sintered at low temperatures, i.e. $1200^{\circ} \mathrm{C}$ and $1300^{\circ} \mathrm{C}$, were good substrates for surface grafting as they had more hydroxyl groups available for the reaction than those sintered at high temperature. However, they had relatively low bending strength which was inadequate for practical applications. There is a trade-off between 


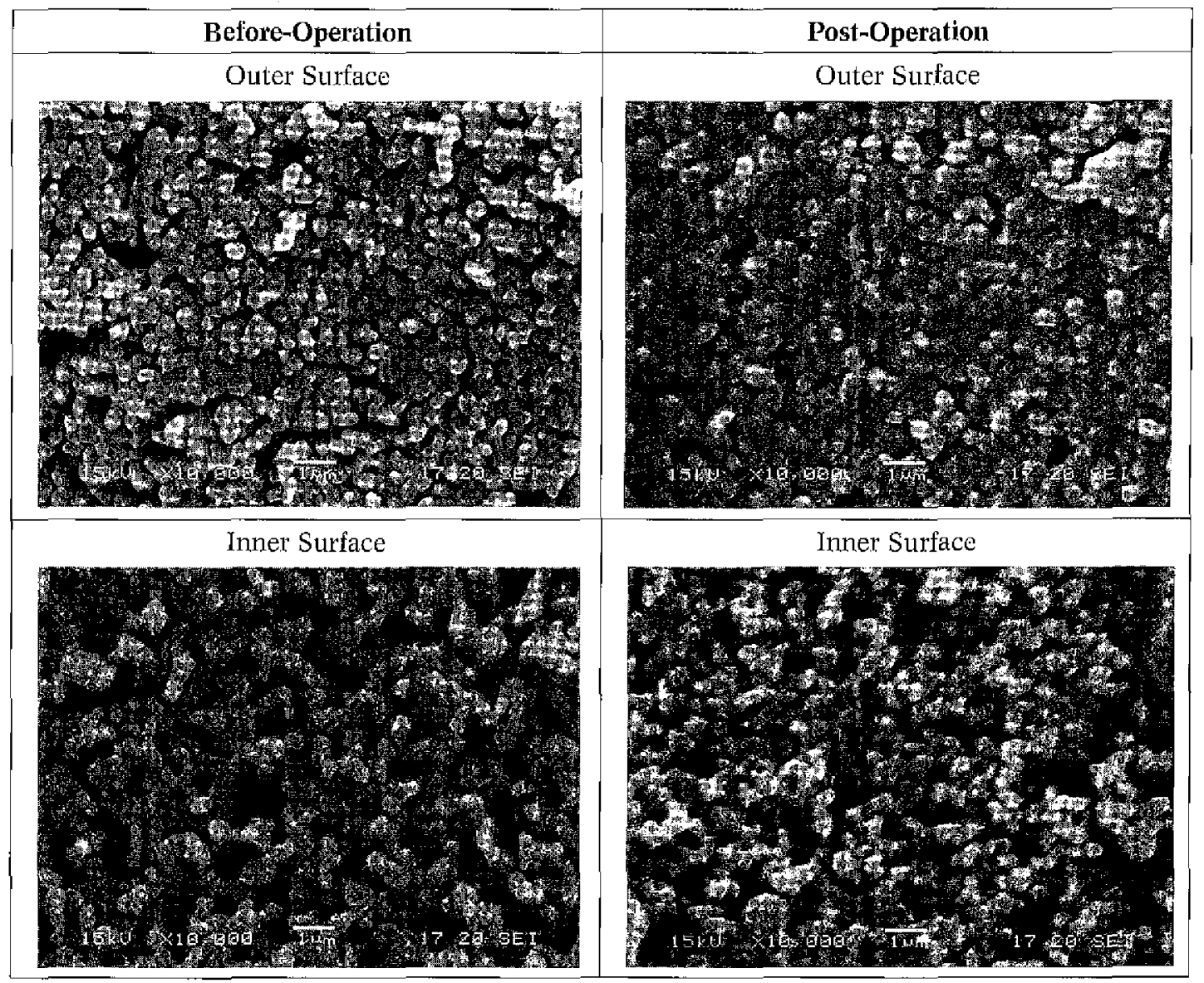

Figure 6 The SEM micrographs of membrane surfaces before and after amine stripping operation

the membrane hydrophobicity and mechanical strength that needs a good balance on them. In this study, only the membranes sintered at $1400^{\circ} \mathrm{C}$ could provide good hydrophobicity and sufficient strength to withstand the condition in an amine stripping column. The mechanical strength is considered to be a weakness that needs to be overcome. The mechanical strength of membranes can be enhanced by several methods. For instance, the elimination of finger-like layers and macrovoids and the promotion of a sponge-like layer in the membrane wall can improve the strength. Another way is the addition of some zirconia particles into alumina spinning suspension to produce a coceramic product, known as zirconia-toughened alumina which is reported to have $30-40 \%$ higher bending strength than pure alumina [18].

\subsection{CONCIUSION}

Surface modification of ceramic membranes can effectively change ceramic membranes from hydrophilic to hydrophobic. The degree of hydrophobicity mainly depends on the reaction between the hydroxyl group on the membrane surface and the FAS molecules. Such coupling reaction is considerably fast that hydrophobic membranes could be prepared within just half an hour of grafting. It was found that the most appropriate grafting period was about 24 hours when most of the hydroxyl groups had been occupied. Beyond this period, the coupling rate was much slower and less efficient. 


\section{REFERENCES}

[1] Gabelman, A. and Hwang, S.-T. 1999. Hollow Fiber Membrane Contactors. J. Membr. Sci. 159(1-2): 61-106.

[2] Alami Younssi, S., Iraqi, A., Rafiq, M., Persin, M., Larbot, A., and Sarrazin, J. 2003. $\gamma$-Alumina Membranes Grafting by Organosilanes and its Application to the Separation of Solvent Mixtures by Pervaporation. Sep. Sci. Technol. 32(1-3): 175-179.

[3] Alami-Younssi, S., Kiefer, C., Larbot, A., Persin, M., and Sarrazin, J. 1998. Grafting $\gamma$-alumina Microporous Membranes by Organosilanes: Characterisation by Pervaporation. $J$. Membr. Sci. 143(1-2): 27-36.

[4] Sah, A., Castricum, H.L., Bliek, A., Blank, D. H. A., and ten Elshof, J. E. 2004. Hydrophobic Modification of $\gamma$-alumina Membranes with Organochlorosilanes. J. Membr. Sci. 243(1-2): 125-132.

[5] Larbot, A., Gazagnes, L., Krajewski, S., Bukowska, M., and Wojciech Kujawski. 2004. Water Desalination using Ceramic Membrane Distillation. Desalination. 168: 367-372.

[6] Akamatsu, Y., Makita, K., Inaba, H., and Minami, T. 2001. Water-repellent Coating Films on Glass Prepared from Hydrolysis and Polycondensation Reactions of Fluoroalkyltrialkoxylsilane. Thin Solid Films. 389(1-2): 138-145.

[7] Picard, C., Larbot, A., Tronel-Peyroz, E., and Berjoan, R. 2004. Characterisation of Hydrophilic Ceramic Membranes Modified by Fluoroalkylsilanes into Hydrophobic Membranes. Solid State Sci. 6(6): 605612.

[8] Picard, C., Larbot, A., Sarrazin, J., Janknecht, P., and Wilderer, P. 2001. Ceramic Membranes for Ozonation in Wastewater Treatment. Ann. Chem. Sci. Mat. 26(2): 13-22.

[9] Krajewski, S. R., Kujawski, W., Dijoux, F., Picard, C., and Larbot, A. 2004. Grafting of $\mathrm{ZrO}_{2}$ Powder and $\mathrm{ZrO}_{2}$ Membrane by Fluoroalkylsilanes. Colloids Surf. A. 243(1-3): 43-47.

[10] Koonaphapdeelert, S. and Li, K. 2007. Preparation and Characterization of Hydrophobic Ceramic Hollow Fibre Membrane. J. Membr. Sci. 291(1-2): 7076.

[11] Koonaphapdeelert, S., Wu, Z., and Li, K. 2009. Carbon Dioxide Stripping in Ceramic Hollow Fibre Membrane Contactors. Chem. Eng. Sci. 64(1): 1-8.

[12] Kong, J. and Li, K. 2001. An Improved Gas Permeation Method for Characterising and Predicting the Performance of Microporous Asymmetric Hollow Fibre Membranes used in Gas Absorption. J. Membr. Sci. 182(1-2): 271-281.

[13] Rulison, C. 1996. Membrane Wettability, in Application Note\#205. Kruss USA. Charlotte, NC.

[14] Barbe, A. M., Hogan, P. A., and Johnson, R. A. 2000. Surface Morphology Changes during Initial Usage of Hydrophobic, Microporous Polypropylene Membranes. $J$. Membr: Sci. 172(1-2): 149-156.

[15] Dindore, V. Y., Brilman, D. W. F., Feron, P. H. M., and Versteeg, G. F. 2004. $\mathrm{CO}_{2}$ Absorption at Elevated Pressures using a Hollow Fiber Membrane Contactor. J. Membr. Sci. 235(1-2): 99-109.

[16] Wang, R., Li, D. F., Zhou, C., Liu, M., and Liang, D. T. 2004. Impact of DEA Solutions with and without $\mathrm{CO}_{2}$ Loading on Porous Polypropylene Membranes Intended for Use as Contactors. J. Membr. Sci. 229(1-2): 147-157.

[17] McElwee, J., Helmy, R., and Fadeev, A. Y. 2005. Thermal Stability of Organic Monolayers Chemically Grafted to Minerals. J. Colloid Interface Sci. 285(2): 551-556.

[18] Wang, J. and Stevens, R, 1989. Zirconiatoughened Alumina (ZTA) Ceramics J. Membr. Sci. 24(10): 3421-3440. 